\title{
Graph-theoretic analysis of multistationarity using degree theory
}

\author{
Carsten Conradi * and Maya Mincheva ${ }^{\dagger}$
}

October 8, 2018

\begin{abstract}
Biochemical mechanisms with mass action kinetics are often modeled by systems of polynomial differential equations (DE). Determining directly if the DE system has multiple equilibria (multistationarity) is difficult for realistic systems, since they are large, nonlinear and contain many unknown parameters. Mass action biochemical mechanisms can be represented by a directed bipartite graph with species and reaction nodes. Graph-theoretic methods can then be used to assess the potential of a given biochemical mechanism for multistationarity by identifying structures in the bipartite graph referred to as critical fragments. In this article we present a graph-theoretic method for conservative biochemical mechanisms characterized by bounded species concentrations, which makes the use of degree theory arguments possible. We illustrate the results with an example of a MAPK network.
\end{abstract}

Keywords. Biochemical mechanisms, mass-action kinetics, multistationarity, bipartite graph, MAPK network.

\section{Introduction}

Biochemical mechanisms of chemical species and elementary reactions are often modeled by differential equations (DE) systems with the species concentrations as variables. Multistability, the existence of multiple stable positive equilibria (for some choice of parameter values) is ubiquitous in models of biochemical mechanisms, such as cell decision [15, 17]. And multistationarity, the existence of multiple positive equilibria is necessary for multistability or a biological switch, a term used in the biological literature.

\footnotetext{
${ }^{*}$ Max Planck Institute Dynamics of Complex Technical Systems, Magdeburg, Germany. Email: conradi@mpi-magdeburg.mpg.de

${ }^{\dagger}$ Corresponding author. Department of Mathematical Sciences, Northern Illinois University, DeKalb, IL 60115, USA. E-mail: mincheva@math.niu.edu
} 
The models in this work will be taken with mass action kinetics resulting in a polynomial right-hand side of the DE system. The DE system models of the biochemical mechanisms of interest are typically high-dimensional, nonlinear and contain many unknown parameters (rate constants and total concentrations). Thus determining parameter values such that multiple equilibria can be found by solving numerically large nonlinear polynomial systems is difficult, if not impossible. On the other hand solving a nonlinear polynomial system with unknown coefficients directly using methods from algebraic geometry has its limitations 14. Therefore other methods and approaches such as graph-theoretic are being developed to answer the question of the existence of multistationarity more easily.

A biochemical mechanism with mass action kinetics can be represented as a directed bipartite graph, which is a graph with two non-intersecting sets of nodes representing species and reactions, and directed edges starting at a species (reaction) node and ending at a reaction (species) node. Graph-theoretic methods can be used to identify structures referred to as critical fragments that are necessary for the existence of multistationarity [16].

Graph-theoretic methods have been used to determine the potential of various biochemical mechanisms for multistationarity $2,7,16,18$. Many of these methods use the one-to-one correspondence between structures in the graph (fragments [16, 18, or cycle structures [7]) and the summands in the determinant of the Jacobian of the right-hand side of the DE system. However, many models have conservation relations with positive coefficients of the species concentrations. The existence of conservation relations results in a non-full rank Jacobian. This leads to considering a coefficient of the characteristic polynomial of the Jacobian different from the constant coefficient and its sign when studying multistationarity. Here we study conservative biochemical mechanisms where all species concentrations participate in at least one conservation relation. This means that all species concentrations are bounded from above and degree theory [10] can be used to study the number of equilibria of the DE model. So far degree theory has been used to study multistationarity in biochemical mechanism models, for example, in [5,8, 9] and graph-theoretic methods have been developed in $2,7,16,18$. Here we combine both approaches to develop a graph-theoretic method for multistationarity in a conservative biochemical mechanism DE model.

This article is organized as follows. Sec. 2 provides an introduction to conservative biochemical mechanisms with mass action kinetics and their properties. In Sec. 3 we discuss consequences of the well-known fact that solutions of the DE systems under study are confined to affine linear subspaces defined by these conservation relations. In Sec. 4 the Jacobian of the original DE system, its parametrization and the determinant of the Jacobian on the level sets is given. In Sec. 5 the degree of a nonlinear function and some of its properties related to a DE system's right-hand side on a given level set is introduced. In Sec. 6 the bipartite graph of a biochemical mechanism with mass action kinetics is introduced. The main result in Sec. 7 (Theorem 4 and Corollary 5) gives a necessary condition for multistationarity for conservative biochemical mechanism models. An example of a MAPK network model studied for multistationarity in [3] is presented in the same 
section.

\section{Preliminaries}

A (bio)chemical mechanism with $n$ species $A_{i}, i=1, \ldots, n$, and $m$ elementary reactions is represented as

$$
\sum_{i=1}^{n} \alpha_{i j} A_{i} \stackrel{k_{j}}{\longrightarrow} \sum_{i=1}^{n} \beta_{i j} A_{i}, \quad j=1 \ldots m,
$$

where $k_{j}>0, j=1, \ldots, m$ are the rate constants. The constants $\alpha_{i j} \geq 0$ and $\beta_{i j} \geq 0$ are small integers called stoichiometric coefficients that account for the number of molecules of species $A_{i}$ participating in the $j^{\text {th }}$ elementary reaction in (1). An example of a chemical mechanism is given below

$$
\begin{aligned}
& A_{2}+A_{3} \stackrel{k_{1}}{\longrightarrow} 2 A_{1}, \\
& A_{3} \stackrel{k_{2}}{\longrightarrow} A_{1} \text {, } \\
& A_{1} \stackrel{k_{3}}{\longrightarrow} A_{3}, \\
& A_{2} \stackrel{k_{4}}{\longrightarrow} A_{1} \\
& A_{1} \stackrel{k_{5}}{\longrightarrow} A_{2} \text {. }
\end{aligned}
$$

Assumption 1. A true reaction is a reaction different from an inflow reaction $A_{i} \rightarrow \emptyset$ or an outflow reaction $\emptyset \rightarrow A_{i}$. An autocatalytic reaction is a reaction of the form $s_{1} A_{i}+\ldots \rightarrow$ $s_{2} A_{i}+\ldots$ where $0<s_{1}<s_{2}$. We assume that every species in (1) is consumed and produced in at least one true non-autocatalytic elementary reaction.

The chemical mechanism in (2) satisfies the above assumption.

We will denote by $x=\left(x_{1}, \ldots, x_{n}\right)$ the vector of concentrations $x_{i}$ of species $A_{i}$ and by $k=\left(k_{1}, \ldots, k_{m}\right)$ the vector of rate constants. If for $y \in \mathbb{R}^{n}, y_{i} \geq 0\left(y_{i}>0\right)$ for all $i$ we will write $y \geq 0(y>0)$. Since each $x_{i} \geq 0$ as a concentration, we have $x \geq 0$. Similarly $k>0$ since each rate constant $k_{j}>0$.

If mass action kinetics is used for the mechanism (1), then the corresponding rate functions are

$$
v_{j}(k, x)=k_{j} x_{1}^{\alpha_{1 j}} \ldots x_{n}^{\alpha_{n j}}, \quad j=1, \ldots, m .
$$

The vector of rate functions will be denoted as $v(k, x)=\left(v_{1}(k, x), \ldots, v_{m}(k, x)\right)$ where $v \geq 0$.

The differential equation (DE) model of a mass-action mechanism such as (1) can be written as

$$
\dot{x}(t)=N v(k, x)=f(k, x)=f(v, x)
$$

where

$$
N_{i j}=\beta_{i j}-\alpha_{i j}
$$


are the entries of the stoichiometric matrix $N$ with dimension $(n \times m)$ and $v(k, x)$ is the vector of rate functions (3). In what follows we will at times interpret the right hand side of (4) as a function of the rate constants $k$ and the species concentrations $x$ and at other times as a function of the reaction rates $v$ and the concentrations $x$, depending on the situation.

For the system (2) we obtain the stoichiometric matrix

$N=\left[\begin{array}{rrrrr}2 & 1 & -1 & 1 & -1 \\ -1 & 0 & 0 & -1 & 1 \\ -1 & -1 & 1 & 0 & 0\end{array}\right]$ and the rate functions $v(k, x)=\left(k_{1} x_{2} x_{3}, k_{2} x_{3}, k_{3} x_{1}, k_{4} x_{2}, k_{5} x_{1}\right)^{T}$.

The equations of the system (4) can be written componentwise as

$$
\dot{x}_{i}(t)=\sum_{j=1}^{m} N_{i j} v_{j}(k, x), \quad i=1, \ldots, n .
$$

The model equations of the mechanism (2) are given below

$$
\begin{aligned}
& \dot{x}_{1}=2 k_{1} x_{2} x_{3}+k_{2} x_{3}-k_{3} x_{1}+k_{4} x_{2}-k_{5} x_{1}=2 v_{1}+v_{2}-v_{3}+v_{4}-v_{5}, \\
& \dot{x}_{2}=-k_{1} x_{2} x_{3}-k_{4} x_{2}+k_{5} x_{1}=-v_{1}-v_{4}+v_{5} \\
& \dot{x}_{3}=-k_{1} x_{2} x_{3}-k_{2} x_{3}+k_{3} x_{1}=-v_{1}-v_{2}+v_{3} .
\end{aligned}
$$

Initially $x(0)=x_{0} \geq 0$, and we will denote a solution $x(t)$ of (4) with initial condition $x_{0}$ as $x\left(t, x_{0}\right)$.

\section{The dynamics on the level sets $\omega_{c_{0}}$}

Let the stoichiometric matrix $N$ have rank $r$. Suppose that at least one solution $\lambda=$ $\left(\lambda_{1}, \ldots, \lambda_{n}\right)$ of the system

$$
\sum_{i=1}^{n} \lambda_{i} N_{i j}=0, \quad j=1, \ldots, m
$$

exists. Then we have $(n-r)$ mass conservation laws

$$
\sum_{i=1}^{n} \lambda_{i} x_{i}=\sum_{i=1}^{n} \lambda_{i} x_{i}(0)
$$

If $\lambda_{i}>0$ for all $i$ in at least one solution $\lambda$, then by (8) it follows that all species concentrations $x_{i}$ are conserved, i.e., $0 \leq x_{i}(t) \leq M$ for all $i$ where $M>0$. A biochemical mechanism (1) with mass-conserved species concentrations will be called conservative biochemical mechanism 8 . 
Assumption 2. Here we study only biochemical mechanisms where the system

$$
\lambda^{T} N=0, \lambda>0
$$

has a solution. As outlined above, in this situation all species concentrations are bounded.

The rank of the stoichiometry matrix $N$ of the system (6) equals 2 . The left kernel of $\mathrm{N}$ is spanned by the vector $\lambda^{T}=(1,1,1)$. Hence the network is conservative and there exists one conservation relation, $x_{1}+x_{2}+x_{3}=$ const.

We can rewrite (8) in a matrix form as

$$
W^{T} x\left(t, x_{0}\right) \equiv W^{T} x_{0}=c_{0}
$$

where $W$ is a full rank $n \times(n-r)$ matrix whose columns span $\operatorname{ker}\left(N^{T}\right)$.

In what follows we interpret the entries of the $(n-r)$ dimensional vector $c_{0}$ as additional parameters and study the dynamics of the system (4) on the level sets

$$
\omega_{c_{0}}=\left\{x \geq 0 \mid W^{T} x=c_{0}\right\} .
$$

This is motivated by the observation that the sets $\omega_{c_{0}}$ are invariant under the dynamics of (4).

Lemma 1 ( $\omega_{c_{0}}$ convex, compact and forward invariant). The set $\omega_{c_{0}}$ is convex, compact and forward invariant.

The proof of Lemma 1 is available in [5].

To study the dynamics of system (4) on invariant sets $\omega_{c_{0}}$ we let $S \in \mathbb{R}^{n \times r}$ be the matrix of full column rank whose columns are an orthonormal basis of $\operatorname{im}(N)$ and we let the matrix $Z \in \mathbb{R}^{n \times(n-r)}$ be the matrix of full column rank whose columns are the orthonormal basis of $\operatorname{im}(N)^{\perp} \equiv \operatorname{ker}\left(N^{T}\right)$. Then the linear transformation

$$
x \rightarrow\left(S^{T} x, Z^{T} x\right)
$$

sends $x \in \mathbb{R}^{n}$ to an element $\xi \in \operatorname{im}(N)$ and to an element $\eta \in \operatorname{im}(N)^{\perp}$ :

$$
\xi:=S^{T} x \text { and } \eta:=Z^{T} x .
$$

Note that $\xi$ and $\eta$ are unique for given $S, Z\left(\right.$ as $\operatorname{im}(N)$ and $\operatorname{im}(N)^{\perp}$ are complementary subspaces). Since by assumption, $S$ and $Z$ are orthonormal we recover

$$
x \equiv x(\xi, \eta)=S \xi+Z \eta .
$$

We further note that by construction $\operatorname{ker}\left(W^{T}\right)=\operatorname{ker}\left(Z^{T}\right)=i m(N)^{\perp}$ and hence all elements $x \in \omega_{c_{0}}$ are sent to the same element $\eta_{0} \in \operatorname{im}(N)^{\perp}$ :

$$
x_{1}, x_{2} \in \omega_{c_{0}} \Rightarrow Z^{T} x_{1}=Z^{T} x_{2}=: \eta_{0}, \forall x_{1}, x_{2} \in \omega_{c_{0}} .
$$


We now apply the linear transformation (11) to the system (4) to obtain:

$$
\begin{aligned}
& \dot{\xi}=S^{T} \dot{x}=S^{T} N v(k, x(\xi, \eta)) \\
& \dot{\eta}=Z^{T} \dot{x}=Z^{T} N v(k, x(\xi, \eta)) \equiv 0 .
\end{aligned}
$$

That is, $\eta$ is constant, reflecting the invariance of $\omega_{c_{0}}$. We introduce the abbreviation:

$$
g_{\eta}(k, \xi):=S^{T} N v(k, x(\xi, \eta)),
$$

where in complete analogy to $c_{0}$ above we interpret $\eta$ as a parameter vector. Then every $\eta_{0} \in \mathbb{R}^{n-r}$ identifies an $r$-dimensional dynamical system

$$
\dot{\xi}=g_{\eta_{0}}(k, \xi) .
$$

Now studying the system (4) restricted to a level set $\omega_{c_{0}}$ is equivalent to studying the system (16) with $\eta_{0}=Z^{T} x_{0}$ for some $x_{0} \in \omega_{c}$.

Solutions $\xi\left(t, \xi_{0}\right)$ of $(16)$ give rise to solutions of (4)

$$
x\left(t, x_{0}\right)=S \xi\left(t, \xi_{0}\right)+Z \eta_{0} .
$$

Since a solution of the system (4), $x\left(t, x_{0}\right) \geq 0$ for all $t \geq 0$, it follows that the corresponding solution $\xi\left(t, \xi_{0}\right)$ of $(16)$ remains in the set

$$
\Omega_{\eta_{0}}=\left\{\xi \in \mathbb{R}^{r} \mid S \xi \geq-Z \eta_{0}\right\} .
$$

The set $\Omega_{\eta_{0}}$ has similar properties as the set $\omega_{c_{0}}$. We have the following lemma for $\Omega_{\eta_{0}}$ which will be used in Corollary 2. The proof is available in [5].

Lemma 2. $\left[\Omega_{\eta_{0}}\right.$ convex, compact and forward invariant] The set $\Omega_{\eta_{0}}$ is convex, compact and forward invariant.

The following lemma compares the number and type of equilibria of (4) in the set $\omega_{c_{0}}$ to the number and type of equilibria of (16) in the set $\Omega_{\eta_{0}}$.

For a set $A$ we will denote its interior by $\operatorname{int}(A)$ and its boundary by $\partial A$.

Lemma 3. [Equilibrium points.]

(a) A positive point $x^{*}$ is an equilibrium of (4) in $\omega_{c_{0}}$ with $c_{0}=W^{T} x^{*}$, if and only if $\xi^{*}=S^{T} x^{*}$ is an equilibrium of (16) for $\eta_{0}=Z^{T} x^{*}$ (where positivity of $x^{*}$ entails $\left.\xi^{*} \in \Omega_{\eta_{0}}\right)$.

(b) The number of equilibria in $\omega_{c_{0}}$ of (4) equals the number of equilibria of (16) in $\Omega_{\eta_{0}}$.

(c) The boundary $\partial \omega_{c_{0}}$ of the set $\omega_{c_{0}}$ contains an equilibrium point of (4) if and only if the boundary $\partial \Omega_{\eta_{0}}$ of $\Omega_{\eta_{0}}$ contains an equilibrium point of (16). 
Proof. (a) This follows since $S^{T} N v\left(k, x^{*}\right)=g_{\eta}\left(k, \xi^{*}\right)=0$, where $x^{*} \in \omega_{c_{0}}$ corresponds to $\xi^{*} \in \Omega_{\eta_{0}}$ such that $\eta_{0}=Z^{T} x^{*}$.

(b) This follows from the fact that $S$ and $Z$ are full rank matrices, and thus the correspondence between $x$ and $(\xi, \eta)$ is one-to-one.

(c) We prove the contrapositive. An equilibrium $x^{*}>0$ of (4) is not on $\partial \omega_{c_{0}}$ if and only if an equilibrium $\xi^{*}$ of $(16)$ is such that $S \xi^{*}>-Z \eta$.

From hereon we will assume level sets $\omega_{c_{0}}$ do not contain equilibria with zero coordinates, referred to as boundary equilibria.

Assumption 3. Here we study only DE models of biochemical mechanisms such that the level set $\omega_{c_{0}}$ does not contain any boundary equilibria, that is,

$$
\text { if } x \in \partial \omega_{c_{0}} \Rightarrow N v(k, x) \neq 0 .
$$

The following corollary follows by Lemma 3 and Assumption 3 .

Corollary 1. Under Assumption 3 the boundary $\partial \Omega_{\eta_{0}}$ of $\Omega_{\eta_{0}}$ does not contain an equilibrium of (16).

The number of equilibria of (4) in $\operatorname{int}\left(\omega_{c_{0}}\right)$ equals the number of equilibria of (16) in int $\left(\Omega_{\eta_{0}}\right)$.

Remark 1. In essence, in Sec.5 we will study the number of equilibria of the reduced system (16) in int $\left(\Omega_{\eta_{0}}\right)$. By Corollary 1 we will obtain the corresponding result on the number of equilibria of the system (4) in $\operatorname{int}\left(\omega_{c_{0}}\right)$.

\section{The Jacobian $J$ parametrized at $(v, x)$ and its projection on $(\xi, \eta)$ space}

The Jacobian matrix $J(k, x)$ of (4) has entries

$$
J_{i l}(k, x)=\sum_{j=1}^{m} N_{i j} \alpha_{l j} k_{j} x_{1}^{\alpha_{1 j}} \ldots x_{l}^{\alpha_{l j}-1} \ldots x_{n}^{\alpha_{n j}} .
$$

Recall (3), then the Jacobian can be written also as

$$
J_{i l}(k, x)=J_{i l}(v, x)=\sum_{j=1}^{m} N_{i j} \alpha_{l j} \frac{v_{j}}{x_{l}} .
$$

Note that if the concentrations $x$ and the rate functions $v(k, x)$ are evaluated at a positive equilibrium, they are positive and can be used as parameters in (19). 
For example, the Jacobian matrix of the right-hand side of the system (6) is

$$
J(v, x)=\left(\begin{array}{ccc}
-\frac{v_{3}}{x_{1}}-\frac{v_{5}}{x_{1}} & \frac{2 v_{1}}{x_{2}}+\frac{v_{4}}{x_{2}} & 2 \frac{v_{1}}{x_{3}}+\frac{v_{2}}{x_{3}} \\
\frac{v_{5}}{x_{1}} & -\frac{v_{1}}{x_{2}}-\frac{v_{4}}{x_{2}} & -\frac{v_{1}}{x_{3}} \\
\frac{v_{3}}{x_{1}} & -\frac{v_{1}}{x_{2}} & -\frac{v_{1}}{x_{3}}-\frac{v_{2}}{x_{3}}
\end{array}\right) .
$$

For the remainder of this contribution we make the following assumption.

Assumption 4. We assume that $\operatorname{im}(J(v, x))=\operatorname{im}(N)$.

Since the rank of $N$ is $r$, it follows under the above assumption that $\operatorname{rank}(J(v, x)) \leq r$ and that the characteristic polynomial of the Jacobian 19 is

$$
\operatorname{det}(\lambda I-J(v, x))=\lambda^{n-r}\left(\lambda^{r}+a_{1} \lambda^{r-1}+\ldots+a_{r-1} \lambda+a_{r}\right)=\lambda^{n-r} q(\lambda),
$$

where the coefficients $a_{i}=a_{i}(v, x), i=1, \ldots, r$ are computed as the sum of all principal minors of order $i$ of the negative Jacobian $-J(v, x)$ [11].

The coefficients $a_{i}(v, x)$ of (21) are rational functions in $x$ and $v$ by $(19)$. For example, the non-zero coefficients of the characteristic polynomial of the Jacobian (20) are

$$
\begin{aligned}
& a_{1}(v, x)=\frac{v_{3}+v_{5}}{x_{1}}+\frac{v_{1}+v_{4}}{x_{2}}+\frac{v_{1}+v_{2}}{x_{3}} \\
& a_{2}(v, x)=\frac{v_{1} v_{3}-v_{1} v_{5}+v_{3} v_{4}}{x_{1} x_{2}}+\frac{-v_{1} v_{3}+v_{1} v_{5}+v_{2} v_{5}}{x_{1} x_{3}}+\frac{v_{1} v_{2}+v_{1} v_{4}+v_{2} v_{4}}{x_{2} x_{3}} .
\end{aligned}
$$

It is easy to verify, that the Jacobian with respect to $\xi$ of $g_{\eta}(k, \xi)$ from 15 is given by

$$
G_{\eta}(v, \xi)=S^{T} J(v, x) S,
$$

where we have suppressed the $\xi, \eta$ dependence of $x$.

The relation between the coefficient $a_{r}(v, x)$ and the determinant of the negative Jacobian $-G_{\eta}(v, \xi)$ of the right-hand side of the reduced system $\dot{\xi}=g(v, \xi)$ is considered in the next lemma. A special case of this lemma with $r=6$ is available in [5].

Lemma 4. The following equivalence is true

$$
\operatorname{det}\left(-G_{\eta}(v, \xi)\right) \equiv \operatorname{det}\left(-S^{T} J(v, x) S\right) \equiv a_{r}(v, x) .
$$

Proof.

Let $\lambda_{1}(v, x), \ldots, \lambda_{n}(v, x)$ be the eigenvalues of $J(v, x)$ and hence the roots of the characteristic polynomial (21), where we assume that $\lambda_{i} \equiv \lambda_{i}(v, x)$. By (21), there exist $(n-r)$ trivial eigenvalues that are identically zero for all values of $(v, x)$ and $r$ non-trivial eigenvalues that are nonzero for some values of $(v, x)$. For simplicity we assume that $\lambda_{i}, i=1, \ldots, r$ are nontrivial and $\lambda_{i}, i=n-r+1, \ldots, n$ are trivial. 
First we show that $a_{r}$ is the product of the nontrivial eigenvalues

$$
a_{r}=\prod_{i=1}^{r} \lambda_{i}
$$

If we write the polynomial $q(\lambda)$ from the characteristic polynomial (21) in factored form and substitute $\lambda=0$ we obtain $a_{r}=\prod_{i=1}^{r} \lambda_{i}$.

Next we show that $\operatorname{det}\left(-S^{T} J(v, x) S\right)=\prod_{i=1}^{r} \lambda_{i}$, which will prove the claim $\operatorname{det}\left(-S^{T} J(v, x) S\right)=$ $a_{r}$. For this purpose we apply the orthonormal transformation $\phi=(S, Z)$ to $J(v, x)$. The transformed matrix has a block form

$$
\phi^{T} J \phi=\left[\begin{array}{c|c}
S^{T} J S & S^{T} J Z \\
\hline 0 & 0
\end{array}\right] .
$$

Since $\phi$ is orthonormal, $J(v, x)$ and $\phi^{T} J(v, x) \phi$ have the same eigenvalues. Both matrices $J(v, x)$ and $\phi^{T} J(v, x) \phi$ have $(n-r)$ trivial eigenvalues. Thus

$$
\operatorname{det}\left(\lambda I-\phi^{T} J(v, x) \phi\right)=\lambda^{n-r} \operatorname{det}\left(\lambda I-S^{T} J(v, x) S\right)=\lambda^{n-r} \tilde{q}(\lambda) .
$$

If $\tilde{q}(\lambda)$ is written in factored form and we let $\lambda=0$ we $\operatorname{obtain} \operatorname{det}\left(-S^{T} J(v, x) S\right)=\prod_{i=1}^{r} \lambda_{i}$. Thus $\operatorname{det}\left(-S^{T} J(v, x) S\right)=a_{r}$.

\section{The degree of $g_{\eta}(k, \xi)$}

Let $U \subset \mathbb{R}^{n}$ be an open and bounded set. The closure of $U$ will be denoted by $\bar{U}$ and the boundary of $U$ by $\partial U$. Thus, $\bar{U}=U \cup \partial U$ is a compact set.

Let $F: \bar{U} \rightarrow \mathbb{R}^{n}$ be a smooth function, where using the usual notation we write $F(x) \in$ $C^{1}(\bar{U})$. We denote the Jacobian matrix of $F(x)$ by

$$
\tilde{J}(x)=\left[\frac{\partial F_{i}}{\partial x_{j}}\right]
$$

and its determinant by $\operatorname{det}(\tilde{J}(x))$. A point $x \in U$ is a regular point for $F(x)$ if $\operatorname{det}(\tilde{J}(x)) \neq 0$. A point $y \in \mathbb{R}^{n}$ is called a regular value if all $x \in U$ such that $F(x)=y$ are regular.

Next we define the (topological or Brouwer) degree of $F(x)$ [10], denoted by $\operatorname{deg}(F)$. In the next definition we use the sign function sign $: \mathbb{R} \rightarrow\{-1,0,1\}$.

Definition 1. [(topological) degree] If $y \notin F(\partial U)$ and $y$ is a regular value, the degree of $F$ is defined by

$$
\operatorname{deg}(F)=\operatorname{deg}(F, U, y)=\sum_{F(x)=y} \operatorname{sign}(\operatorname{det}(-\tilde{J}(x))) .
$$


Remark 2. Note that we use $\operatorname{sign}(\operatorname{det}(-\tilde{J}(x)))$ in 26$)$ in place of $\operatorname{sign}(\operatorname{det}(\tilde{J}(x)))$ to avoid the case of the degree depending on the dimension $n$ of $\mathbb{R}^{n}$ similarly to [13].

The sum in 26 is over all solutions $x \in U$ of $F(x)=y$ such that $\operatorname{det}(-\tilde{J}(x)) \neq 0$. If $F(x)=y$ does not have solutions $x \in U$, then we set $\operatorname{deg}(F)=0$. Since we are interested in the equilibrium solutions $x^{*}$ of $\dot{x}=F(x)$ that satisfy $F\left(x^{*}\right)=0$, we will let $y=0$ in (26).

Next we study the degree of the function $g_{\eta}(v, \xi)$ defined in $(16)$. Recall that $x \equiv x(\eta, \xi)$ and that $a_{r}(v, x)=\operatorname{det}\left(-G_{\eta}(v, \xi)\right)$ by Lemma 4 , where $G_{\eta}(v, \xi)$ is the Jacobian of the function $g_{\eta}(v, \xi)$ given in $(24)$.

The next lemma is similar to Lemma 5.4 available in the Supporting Information of [5].

Remark 3. Note that in the lemma and corollaries below the assumption that $\partial \Omega_{\eta}$ does not contain any boundary equilibrium of the system (16) is automatically satisfied by Assumption 3 and Corollary 1 .

Lemma 5. Let $g_{\eta}$ be as in (15). Fix $\eta \in \mathbb{R}^{n-r}$ and assume that the boundary $\partial \Omega_{\eta}$ does not contain any equilibria of (16). If all equilibria $\xi \in \Omega_{\eta}$ are regular, then

$$
\operatorname{deg}\left(g_{\eta}, \operatorname{int}\left(\Omega_{\eta}\right), 0\right)=\sum_{\left\{\xi \in \operatorname{int}\left(\Omega_{\eta}\right) \mid g_{\eta}(\xi)=0\right\}} \operatorname{sign}\left(a_{r}(v, x(\eta, \xi))\right) .
$$

Remark 4. By Corollary 1 the equilibria of (16) are in $\operatorname{int}\left(\Omega_{\eta}\right)$. Therefore the degree of $g_{\eta}(\xi), \operatorname{deg}\left(g_{\eta}, \operatorname{int}\left(\Omega_{\eta}\right), 0\right)$ given by (27) is well defined.

We obtain the following corollaries on the degree of $g_{\eta}(k, \xi)$ where $\xi \in \Omega_{\eta}$. Similar corollaries for the special case of $r=6$ are available in 5 .

First we need the following theorem on the homotopy invariance of the degree [8].

Theorem 1. Let $U \subset \mathbb{R}^{n}$ be a bounded and open set. Let $H(x, s): \bar{U} \times[0,1] \rightarrow \mathbb{R}^{n}$, be a continuously varying set of functions such that $H(x, s)$ does not have any zeroes on the boundary of $U$ for all $s \in[0,1]$. Then $\operatorname{deg}(H(x, s))$ is constant for all $s \in[0,1]$.

Corollary 2. Let $g_{\eta}$ be as in (15) and assume that the boundary $\partial \Omega_{\eta}$ does not contain any equilibria of (16). Then the the following holds true:

$$
\operatorname{deg}\left(g_{\eta}, \operatorname{int}\left(\Omega_{\eta}\right), 0\right)=1 .
$$

Proof. Since $f(k, x)$ is smooth on $\omega_{c_{0}}$, therefore $g_{\eta}(k, \xi)$ is smooth on $\Omega_{\eta}$. Let $k$ be fixed but arbitrary so that $g_{\eta}(k, \xi)=g_{\eta}(\xi)$.

We have $\Omega_{\eta}=\operatorname{int}\left(\Omega_{\eta}\right) \cup \partial \Omega_{\eta}$, where $\operatorname{int}\left(\Omega_{\eta}\right)$ is the interior of $\Omega_{\eta}$ and $\partial \Omega_{\eta}$ is the boundary of $\Omega_{\eta}$. By Lemma 2 , $\operatorname{int}\left(\Omega_{\eta}\right)$ is bounded. We follow the proof of $\left[9\right.$, Lemma 2]. Let $\bar{\xi} \in \operatorname{int}\left(\Omega_{\eta}\right)$ be an arbitrary point and consider the function

$$
G(\xi)=\bar{\xi}-\xi
$$


By Definition 1 it follows that

$$
\operatorname{deg}\left(G, \operatorname{int}\left(\Omega_{\eta}\right), 0\right)=1 .
$$

Next we show that $g_{\eta}$ and $G$ are homotopic. We define the following homotopy

$$
H(\xi, s)=s g_{\eta}(\xi)+(1-s) G(\xi)
$$

where $0 \leq s \leq 1$. Therefore $H(\xi, s)$ is continuous on $\Omega_{\eta} \times[0,1], H(\xi, 0)=G(\xi)$ and $H(\xi, 1)=g_{\eta}(\xi)$. To apply Theorem 1 we need to show that $H(\xi, s) \neq 0$ for all $\xi \in \partial \Omega_{\eta}$ and for all $s \in[0,1]$. The latter is true if $s=0$ since $\bar{\xi} \in \operatorname{int}\left(\Omega_{\eta}\right)$ and if $s=1$ by Corollary 1 . Suppose it is not true if $s \in(0,1)$, then there exists $\tilde{\xi} \in \partial \Omega$ and $\tilde{s} \in(0,1)$ such that

$$
g_{\eta}(\tilde{\xi})=-\frac{1-s}{s} G(\tilde{\xi}) .
$$

By the convexity of $\Omega_{\eta}$ it follows that $G(\xi)$ points strictly inwards at $\xi=\tilde{\xi}$. Thus $g_{\eta}(\xi)$ at $\xi=\tilde{\xi}$ points strictly outwards. This is a contradiction since $\Omega_{\eta}$ is forward invariant by Lemma 2. Thus the claim in (28) follows by equation (29) since the degree is homotopy invariant by Theorem 1 .

Corollary 3. Let $\eta$ and $k$ be given and note that $v \equiv v(k, x)$. Assume that the boundary $\partial \Omega_{\eta}$ does not contain any equilibria of (16). If $a_{r}(v, x(\eta, \xi))>0$ for all $\xi \in \operatorname{int}\left(\Omega_{\eta}\right)$, then the equation

$$
g_{\eta}(v, \xi)=0, \xi \in \Omega_{\eta}
$$

has a unique solution.

If all solutions of $g_{\eta}(v, \xi)=0, \xi \in \operatorname{int}\left(\Omega_{\eta}\right)$ are regular, then the number of solutions in $\operatorname{int}\left(\Omega_{\eta}\right)$ is odd.

Proof. Recall that by Lemma 4, $\operatorname{det}\left(-G_{\eta}\right)=a_{r}(v, x(\eta, \xi))$. Suppose that $a_{r}(v, x(\eta, \xi))>0$ for all $\xi \in \operatorname{int}\left(\Omega_{\eta}\right)$. Then by Corollary $2, \operatorname{deg}\left(g_{\eta}, \operatorname{int}\left(\Omega_{\eta}\right), 0\right)=1$. Thus by (27), it follows that the number of equilibria in $\operatorname{int}\left(\Omega_{\eta}\right)$ equals one.

If all equilibria $\xi^{*}$ are regular, then $a_{r}(v, x(\eta, \xi))$ is either positive or negative at $\xi=\xi^{*}$. Since $\operatorname{deg}\left(g_{\eta}, \operatorname{int}\left(\Omega_{\eta}\right), 0\right)=1$ by Corollary 2 , it follows by (27) that the number of equilibria has to be odd.

Now we turn to the system (4). In the next theorem we show that the system (4) has an interior equilibrium solution in any set $\omega_{c_{0}}$ where $c_{0}$ is fixed.

Theorem 2. Let $f(k, x)$ be as in (4) and recall Assumption 3. If

$$
a_{r}(v, x)>0 \text {, for all } v>0 \text { and } x>0 \text {, }
$$

then

$$
f(k, x)=0, x \in \omega_{c_{0}}
$$

has a unique solution for all $c_{0}>0$. 


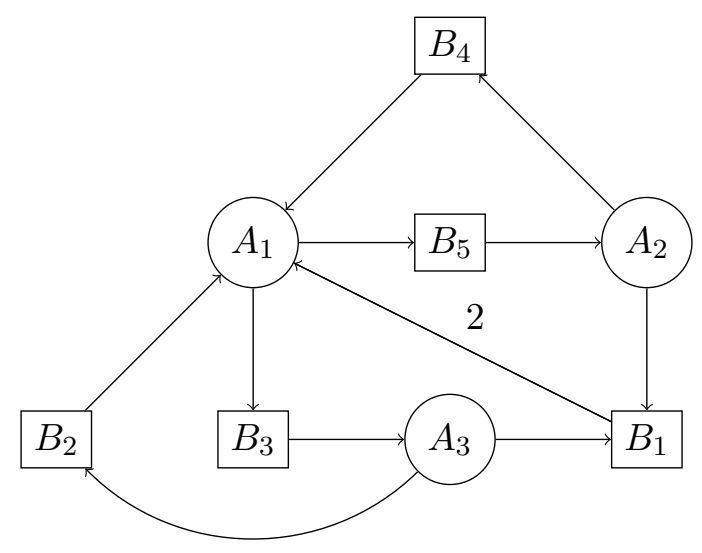

Figure 1: Bipartite graph of the reaction mechanism (2).

Proof. Pick $c_{0}>0$ and choose any $x_{0} \in \omega_{c_{0}}$. Compute $\xi_{0}=S^{T} x_{0}, \eta_{0}=Z^{T} x_{0}$. By assumption

$$
a_{r}\left(v, x\left(\eta_{0}, \xi\right)\right)>0, \forall v>0 \text { and } \forall \xi \in \Omega_{\eta_{0}} .
$$

Thus, by Corollary $3, g_{\eta_{0}}(k, \xi), \xi \in \Omega_{\eta_{0}}$ has a unique solution $\xi^{*}$. And by Lemma 3 (a)-(b), an equilibrium $x^{*}=S \xi^{*}+Z \eta_{0}$ of $\dot{x}=f\left(k, x^{*}\right), x^{*} \in \operatorname{int}\left(\omega_{c_{0}}\right)$ is unique.

\section{The bipartite digraph of a biochemical mechanism}

For the convenience of the reader, in this section we present definitions regarding the bipartite digraph of a biochemical mechanism (1) [16, 18]. To illustrate the definitions we will continue to use as an example the mechanism (2).

A directed bipartite graph (bipartite digraph) has a node set that consists of two disjoint subsets, $V_{1}$ and $V_{2}$, and each of its directed edges (arcs) has one end in $V_{1}$ and the other in $V_{2}[12]$.

The bipartite digraph $G$ of a biochemical reaction network (1) is defined as follows. The nodes are separated into two sets, one for the chemical species $V_{1}=\left\{A_{1}, A_{2}, \ldots, A_{n}\right\}$ and one for the elementary reactions $V_{2}=\left\{B_{1}, B_{2}, \ldots, B_{m}\right\}$. We draw an arc from $A_{k}$ to $B_{j}$ if and only if species $A_{k}$ is a reactant in reaction $j$, i.e., if the stoichiometric coefficient $\alpha_{k j}>0$ in (1). Similarly, we draw an arc from $B_{j}$ to $A_{i}$ if and only if $A_{i}$ is a product in reaction $j$, i.e., if the stoichiometric coefficient $\beta_{i j}>0$ in (1). Therefore the set of arcs $E(G)$ consists of arcs such as $\left(A_{k}, B_{j}\right)$ and $\left(B_{j}, A_{i}\right)$. Hence the bipartite digraph can be defined as $G=\{V, E(G)\}$ where $V=V_{1} \cup V_{2}$ is the set of nodes and $E(G)$ is the set of arcs. If an arc is not weighted explicitly, we assume that its weight equals 1 . The corresponding bipartite digraph of the mechanism (2) is shown in Figure 1. 


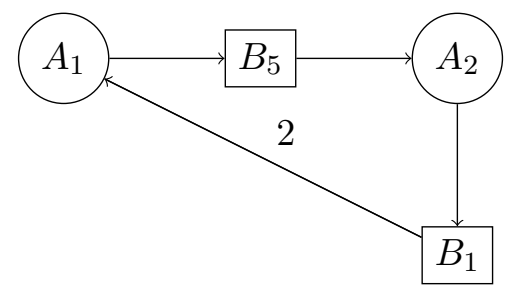

Figure 2: Cycle $C\left(\begin{array}{l}A_{1}, A_{2} \\ B_{5}, B_{1}\end{array}\right)$ of the bipartite graph of the reaction mechanism 2 .

The element $\left[A_{k}, B_{j}\right]$ is an edge if $\alpha_{k j}>0$, i.e., if species $A_{k}$ is a reactant in reaction $j$. The weight of an edge $E=\left[A_{k}, B_{j}\right]$ is defined as

$$
K_{E}=-\alpha_{k j}^{2}
$$

For example, the edge $E=\left[A_{1}, B_{5}\right]$ corresponding to the arc $\left(A_{1}, B_{5}\right)$ in Figure 1 has weight $K_{E}=-1$.

If $\alpha_{k j} \beta_{i j}>0$, then the $\operatorname{arcs}\left(A_{k}, B_{j}\right)$ and $\left(B_{j}, A_{i}\right)$ form a positive path $\left[A_{k}, B_{j}, A_{i}\right]$ that corresponds to the production of $A_{i}$ from $A_{k}$ in a reaction $j$. The weight of the positive path $\left[A_{k}, B_{j}, A_{i}\right]$ is defined as $\alpha_{k j} \beta_{i j}$. For example, the positive path $\left[A_{1}, B_{5}, A_{2}\right]$ in Figure 1 has weight 1.

If $\alpha_{k j} \alpha_{i j}>0$, then the $\operatorname{arcs}\left(A_{k}, B_{j}\right)$ and $\left(A_{i}, B_{j}\right)$ form a negative path $\left[\overline{A_{k}, B_{j}, A_{i}}\right]$ that corresponds to $A_{k}$ and $A_{i}$ interacting as reactants in reaction $j$. The weight of the negative path $\left[\overline{A_{k}, B_{j}, A_{i}}\right]$ is defined as $-\alpha_{k j} \alpha_{i j}$. Note that the negative paths $\left[\overline{A_{k}, B_{j}, A_{i}}\right]$ and $\left[\overline{A_{i}, B_{j}, A_{k}}\right]$ are considered to be different since they start at a different species node. For example, both $\left[\overline{A_{2}, B_{1}, A_{3}}\right]$ and $\left[\overline{A_{3}, B_{1}, A_{2}}\right]$ in Figure 1 are negative paths with weight -1 . We note that the direction of the arcs is followed in the positive paths but not in the negative paths.

A cycle $C$ of $G$ is a sequence of distinct paths with the last species node of each path being the same as the first species node of the next path $C=\left\{\left(A_{i_{1}}, B_{j_{1}}, A_{i_{2}}\right),\left(A_{i_{2}}, B_{j_{2}}, A_{i_{3}}\right), \ldots\right.$, $\left.\left(A_{i_{k-1}}, B_{j_{k-1}}, A_{i_{k}}\right),\left(A_{i_{k}}, B_{j_{k}}, A_{i_{1}}\right)\right\}$. A cycle will be denoted by $C=\left(\begin{array}{l}A_{i_{1}}, A_{i_{2}}, \ldots, A_{i_{k}} \\ B_{j_{1}}, B_{j_{2}}, \ldots, B_{j_{k}}\end{array}\right)$, where the number of species nodes defines its order. The set of species nodes in a cycle is distinct, but there may be a repetition among the reaction nodes. This is because negative paths containing the same nodes are considered different depending on the starting species node. For example, $C=\left(\begin{array}{c}A_{2}, A_{3} \\ B_{1}, B_{1}\end{array}\right)$ in Figure 1 is a cycle formed by the two negative paths $\left[\overline{A_{2}, B_{1}, A_{3}}\right]$ and $\left[\overline{A_{3}, B_{1}, A_{2}}\right]$.

A cycle is positive if it contains an even number of negative paths and negative if it contains an odd number of negative paths. The sign of a cycle $C$ can also be determined by the cycle weight which is a product of all corresponding weights of negative and positive 
paths of $C$

$$
K_{C}=\prod_{\overline{\left[A_{k}, B_{j}, A_{i}\right]} \in C}\left(-\alpha_{k j} \alpha_{i j}\right) \prod_{\left[A_{k}, B_{j}, A_{i}\right] \in C} \alpha_{k j} \beta_{i j} .
$$

For example, $C=\left(\begin{array}{l}A_{1}, A_{2} \\ B_{5}, B_{1}\end{array}\right)$ in Figure 2 is a positive cycle of order 2 with weight $K_{C}=2$.

A subgraph $g=\left\{\mathrm{L}_{1}, \mathrm{~L}_{2}, \ldots, \mathrm{L}_{s}\right\}$ of $G$ consists of edges or cycles $\mathrm{L}_{i}, i=1, \ldots, s$, where each species is the beginning of only one edge, or one path participating in a cycle. The number of species nodes in a subgraph is defined as its order. The subgraph weight is defined as

$$
K_{g}=(-1)^{c} \prod_{C \in g} K_{C} \prod_{E \in g}\left(-K_{E}\right)
$$

where $c$ is the number of cycles in $g, K_{C}$ is the cycle weight (31) and $K_{E}$ is the edges weights (30) of the cycles and edges in $g$. For example, the subgraph $g=\left\{\left[A_{3}, B_{2}\right],\left(\begin{array}{c}A_{1}, A_{2} \\ B_{1}, B_{5}\end{array}\right)\right\}$ with weight $K_{g}=-2$ is shown in Figure 3 .

Since more than one path can exist between species nodes via different reaction nodes in a bipartite digraph, the number of subgraphs through the same node sets may be greater than one. The set of all subgraphs $g$ of order $k$ with the same species nodes $\bar{V}_{1}=\left\{A_{i_{1}}, \ldots A_{i_{k}}\right\}$ and reaction nodes $\bar{V}_{2}=\left\{B_{j_{1}}, \ldots B_{j_{k}}\right\}$ sets is called a fragment of order $k$ and is denoted by $S_{k}\left(\begin{array}{l}i_{1}, \ldots, i_{k} \\ j_{1}, \ldots, j_{k}\end{array}\right)$. For a fragment $S_{k}\left(\begin{array}{l}i_{1}, \ldots, i_{k} \\ j_{1}, \ldots, j_{k}\end{array}\right)$ we define the number

$$
K_{S_{k}}=\sum_{g \in S_{k}} K_{g}
$$

as the fragment weight. If $K_{S_{k}}<0$, then $S_{k}$ is defined as a critical fragment.

For example, the fragment $S_{2}\left(\begin{array}{l}1,2 \\ 5,1\end{array}\right)$ shown in Figure 4 together with its two subgraphs $g_{1}=C_{2}=\left(\begin{array}{l}A_{1}, A_{2} \\ B_{5}, B_{1}\end{array}\right)$ and $g_{2}=\left\{\left[A_{1}, B_{5}\right],\left[A_{2}, B_{1}\right]\right\}$. The first subgraph $g_{1}$ is a positive cycle and thus it has a negative weight. Therefore $S_{2}\left(\begin{array}{l}1,2 \\ 5,1\end{array}\right)$ is a critical fragment since

$$
K_{S_{2}}=\sum_{g \in S_{2}} K_{g}=K_{g_{1}}+K_{g_{2}}=-2+1=-1<0
$$

In [16, 18] it is shown that the coefficients of the characteristic polynomial (21) have the following graph-theoretic representation.

Theorem 3. A coefficient of the characteristic polynomial of the Jacobian (19) can be written as

$$
a_{k}(v, x)=\sum_{S_{k}\left(\begin{array}{c}
i_{1}, \ldots, i_{k} \\
j_{1}, \ldots, j_{k}
\end{array}\right)} K_{S_{k}} \frac{v_{j_{1}} \ldots v_{j_{k}}}{x_{i_{1}} \ldots x_{i_{k}}}, \quad k=1, \ldots, n .
$$

where $S_{k}\left(\begin{array}{l}i_{1}, \ldots, i_{k} \\ j_{1}, \ldots, j_{k}\end{array}\right)$ is a fragment of order $k$ and $K_{S_{k}}$ is the fragment's weight. 


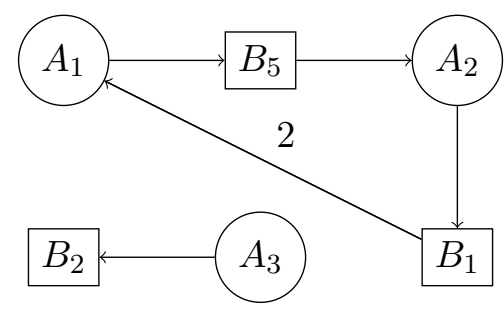

Figure 3: Subgraph $g=\left\{\left[A_{3}, B_{2}\right],\left(\begin{array}{c}A_{1}, A_{2} \\ B_{5}, B_{1}\end{array}\right)\right\}$ of the bipartite graph of the reaction mechanism (2).
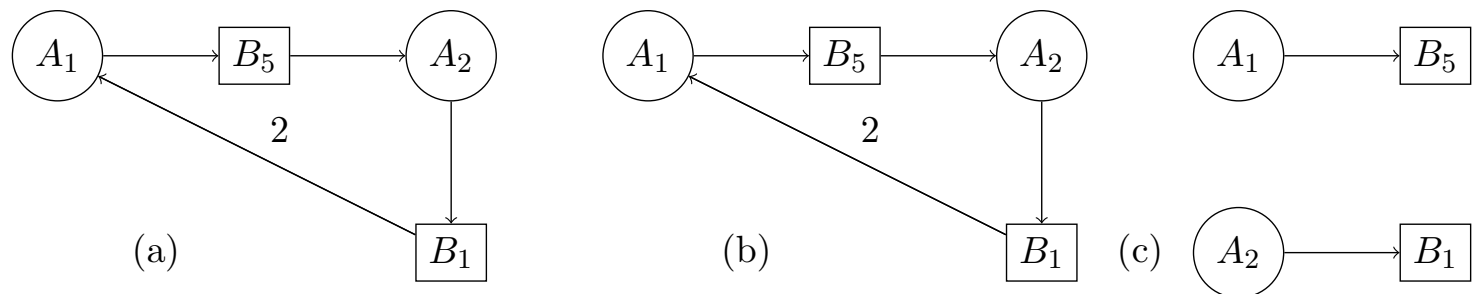

Figure 4: The critical fragment $S_{2}\left(\begin{array}{l}1,2 \\ 5,1\end{array}\right)$ (shown in (a)) together with its two subgraphs $\left.g_{1}=C_{2}=\left(\begin{array}{l}A_{1}, A_{2} \\ B_{5}, B_{1}\end{array}\right)\right\}$ (shown in (b))and $g_{2}=\left\{\left[A_{1}, B_{5}\right],\left[A_{2}, B_{1}\right]\right\}$ (shown in (c)).

Note that similar terms in $a_{k}(v, x)$ have been combined using summation over the subgraphs of a fragment (33), and (34) is in a simplified form. It follows by (34) that the correspondence between a fragment $S_{k}\left(\begin{array}{l}i_{1}, \ldots, i_{k} \\ j_{1}, \ldots, j_{k}\end{array}\right)$ and a non-zero term in $a_{k}(v, x)$ is one-toone. For example, the first negative term in the coefficient (23) corresponds to the critical fragment $S_{2}\left(\begin{array}{l}1,2 \\ 5,1\end{array}\right)$ shown in Figure 4.

The next corollary follows immediately by Theorem 3 .

Corollary 4. Recall the function $f$ from (4) with Jacobian $J$ as in (19). The last (not identically zero) coefficient of the characteristic polynomial (21) can be written as

$$
a_{r}(v, x)=\sum_{S_{r}\left(\begin{array}{c}
i_{1}, i_{2}, \ldots, i_{r} \\
j_{1}, j_{2}, \ldots j_{r}
\end{array}\right) \in G} K_{S_{r}} \frac{v_{j_{1}} \ldots v_{j_{r}}}{x_{i_{1}} \ldots x_{i_{r}}},
$$

where $r$ is the rank of the stoichiometric matrix $N$.

Remark 5. Recall that $x \equiv x(\nu, \xi)$. Hence, for fixed values of $\eta$, one may consider $a_{r}(v, x)$ as a function of $\xi$. We further note that $a_{r}(v, x)$ depends on $k$, as $v \equiv v(k, x)$. 


\section{Multistationarity}

This section contains the main result of the paper. First we will show that the existence of multistationarity requires a negative term in $a_{r}(v, x)$ given by (35). Thus a critical fragment of order $r$ corresponding uniquely to a negative term in $a_{r}(v, x)$ must be present in the bipartite graph of a conservative biochemical mechanism model showing multistationary.

We have that the coefficient $a_{r}(v, x)$ contains at least one positive term that corresponds to a product of positive diagonal entries of the negative Jacobian $-J(x, v)$. This is true since we assumed (Sec. 2, Assumption 1) that each species participates as a reactant (and a product) in at least one reaction.

Theorem 4. Recall the function $g_{\eta}$ from (15). Let $\eta$ and $k$ be given such that $g_{\eta}(k, \xi)=0$, $\xi \in \Omega_{\eta}$ has more that one solution. Under Assumption 3, if all solutions are regular, then $a_{r}(v, x)$ contains a negative term.

Proof. Suppose not, i.e., $a_{r}(v, x)$ contains only positive terms. Then $a_{r}(v, x)>0$ for any $k>0$ and $\xi \in \Omega_{\eta}$ (recall that $v \equiv v(k, x)$ and $\left.x \equiv x(\eta, \xi)\right)$. This is in contradiction with Corollary 3 . Therefore $a_{r}(v, x)$ contains at least one negative term.

Corollary 5. In the setting of Theorem 4, if the system $g_{\eta}(k, \xi)=0, \xi \in \Omega_{\eta}$ has multiple solutions, then the bipartite graph of the conservative biochemical mechanism (1) contains a critical fragment $S_{r}$, where $r$ is the rank of the stoichiometric matrix $N$.

Proof. This follows by Theorem 4, Corollary 4 and the one-to-one correspondence between a negative term in $a_{r}(v, x)$ and a critical fragment.

Example. The biochemical mechanism (2) is conservative since its concentrations satisfy the conservation relation $x_{1}+x_{2}+x_{3}=c_{0}$. The system (6) has no boundary equilibria since $(0,0,0)$ does not satisfy the conservation relation $x_{1}+x_{2}+x_{3}=c_{0}$. Thus the graphtheoretic condition developed here applies.

The existence of multistationarity requires a critical fragment of order equal to the rank of the stoichiometric matrix by Corollary 5. Two critical fragments of order two, the rank of the stoichiometric matrix of (2), exist in the bipartite graph of the mechanism (2) shown in Figure 1. The first critical fragment $S_{2}\left(\begin{array}{l}1,2 \\ 5,1\end{array}\right)$ is shown in Figure 4 . The second critical fragment $S_{2}\left(\begin{array}{l}1,3 \\ 3,1\end{array}\right)$ is similar in structure - $S_{2}\left(\begin{array}{l}1,3 \\ 3,1\end{array}\right)$ contains a subgraph which is a positive cycle of order $2, g_{1}=C\left(\begin{array}{c}A_{1}, A_{3} \\ B_{3}, B_{1}\end{array}\right)$ and a subgraph of edges $g_{2}=\left\{\left[A_{1}, B_{3}\right],\left[A_{3}, B_{1}\right]\right\}$. Therefore, the existence of multiple (always an odd number) regular equilibria of (6) in the interior of a level set $x_{1}+x_{2}+x_{3}=c_{0}$ for some $c_{0}$ is possible, for some values of the rate constants $k$.

Example. The MAPK network belongs to a family of biochemical networks known as MAPK cascades that have been extensively studied in recent years [4, 6, 15. A mass action kinetics MAPK model with a single layer is studied in [3]. We use the proposed 
here graph-theoretic method to analyze the MAPK network for multistationarity. We find critical fragments in the bipartite graph of the MAPK network, that are responsible for the already discovered multistationarity in $3,5$. .

We will use $A$ for either a MAPKK or a MAPK, $E_{1}$ for mono-phosphorylated MAPKKK or double-phosphorylated MAPKK and $E_{2}$ for MAPKK'ase or MAPK'ase. The biochemical mechanism involves the species $A, A_{p}, A_{p p}, E_{1}, E_{2}, A E_{1}, A_{p} E_{1}, A_{p p} E_{2}$, and $A_{p} E_{2}$ and the 12 elementary reactions

$$
\begin{gathered}
A+E_{1} \underset{k_{2}}{\stackrel{k_{1}}{\rightleftharpoons}} A E_{1} \stackrel{k_{3}}{\longrightarrow} A_{p}+E_{1} \underset{k_{5}}{\stackrel{k_{4}}{\rightleftharpoons}} A_{p} E_{1} \stackrel{k_{6}}{\longrightarrow} A_{p p}+E_{1} \\
A_{p p}+E_{2} \underset{k_{8}}{\stackrel{k_{7}}{\rightleftharpoons}} A_{p p} E_{2} \stackrel{k_{9}}{\longrightarrow} A_{p}+E_{2} \underset{k_{11}}{\stackrel{k_{10}}{\rightleftharpoons}} A_{p} E_{2} \stackrel{k_{12}}{\longrightarrow} A+E_{2} .
\end{gathered}
$$

Let each species in (36) be associated with a continuously differentiable variable representing its concentration. The concentration variables are chosen as follows: $x_{1}$ for $A, x_{2}$ for $E_{1}, x_{3}$ for $A E_{1}, x_{4}$ for $A_{p}, x_{5}$ for $A_{p} E_{1}, x_{6}$ for $A_{p p}, x_{7}$ for $E_{2}, x_{8}$ for $A_{p p} E_{2}$ and $x_{9}$ for $A_{p} E_{2}$. The following system of ordinary differential equations is obtained as a model of (36) with mass action kinetics

$$
\begin{aligned}
& \dot{x}_{1}=-k_{1} x_{1} x_{2}+k_{2} x_{3}+k_{12} x_{9} \\
& \dot{x}_{2}=-k_{1} x_{1} x_{2}+\left(k_{2}+k_{3}\right) x_{3}-k_{4} x_{2} x_{4}+\left(k_{5}+k_{6}\right) x_{5} \\
& \dot{x}_{3}=k_{1} x_{1} x_{2}-\left(k_{2}+k_{3}\right) x_{3} \\
& \dot{x}_{4}=k_{3} x_{3}-k_{4} x_{2} x_{4}+k_{5} x_{5}+k_{9} x_{8}-k_{10} x_{4} x_{7}+k_{11} x_{9} \\
& \dot{x}_{5}=k_{4} x_{2} x_{4}-\left(k_{5}+k_{6}\right) x_{5} \\
& \dot{x}_{6}=k_{6} x_{5}-k_{7} x_{6} x_{7}+k_{8} x_{8} \\
& \dot{x}_{7}=-k_{7} x_{6} x_{7}+\left(k_{8}+k_{9}\right) x_{8}-k_{10} x_{4} x_{7}+\left(k_{11}+k_{12}\right) x_{9} \\
& \dot{x}_{8}=k_{7} x_{6} x_{7}-\left(k_{8}+k_{9}\right) x_{8} \\
& \dot{x}_{9}=k_{10} x_{4} x_{7}-\left(k_{11}+k_{12}\right) x_{9}
\end{aligned}
$$

Since the total concentrations of $E_{1}, E_{2}$ and $A$ are constant, three conservation relations exist

$$
\begin{aligned}
x_{2}+x_{3}+x_{5} & =c_{1}, \\
x_{7}+x_{8}+x_{9} & =c_{2} \\
x_{1}+x_{3}+x_{4}+x_{5}+x_{6}+x_{8}+x_{9} & =c_{3} .
\end{aligned}
$$

where each $c_{i}>0, i=1,2,3$. Thus 38 can be written as $\widetilde{W}^{T} x=c_{0}$ where

$$
\widetilde{W}^{T}=\left[\begin{array}{lllllllll}
0 & 1 & 1 & 0 & 1 & 0 & 0 & 0 & 0 \\
0 & 0 & 0 & 0 & 0 & 0 & 1 & 1 & 1 \\
1 & 0 & 1 & 1 & 1 & 1 & 0 & 1 & 1
\end{array}\right] .
$$


Correspondingly the level set of the system (37) is

$$
\widetilde{\omega}_{c_{0}}=\left\{x \geq 0 \mid \widetilde{W}^{T} x=c_{0}\right\} .
$$

Since the MAPK network (36) is conservative by (38), the theory developed here applies to it, provided that the model system (37) does not have any boundary equilibrium (equilibrium with at least one zero coordinate) in $\tilde{\omega}_{c_{0}}$. The following lemma is part of Lemma 3.1 in the Supporting information of [5].

Lemma 6. The set $\widetilde{\omega}_{c_{0}}$ contains no boundary equilibria of the system (37).

The bipartite graph of the MAPK network (36) is shown in Figure 5.

The necessary condition for multistationarity requires the existence of a critical fragment of order equal to the rank of the stoichiometric matrix by Corollary 5 . Since the rank of the stoichiometric matrix for the MAPK network (36) equals 6, using the package GraTeLPy we have enumerate all critical fragments of order 6 in [19]. The 9 critical fragments of order 6 of the MAPK network are shown in Figure 6 and Figure 7. Therefore, the existence of multiple (an odd number) regular equilibria of the system (37) in the interior of a level set (40) for some $c_{0}$ is possible for some values of the rate constants $k$. In fact we show in 55 that for some given values of the rate constants $k$, three equilibria in $38 \mathrm{a}$ - 38c exist where $c_{0}$ is chosen based on Corollary 1 in $[5]$.

\section{Discussion}

We have studied conservative biochemical mechanisms characterized by bounded concentrations of all their species. We have obtained a graph-theoretic condition for multistationarity for conservative biochemical mechanisms with mass action kinetics. In essence the graph-theoretic condition is the same as for non-conservative biochemical mechanisms

- the existence of a critical fragment of order $r$, the rank of the stoichiometric matrix, is required for multistationarity. The difference between the case of conservative and nonconservative biochemical mechanisms is that, in the first case we can apply degree theory arguments 10 and in the second only bifurcation theory can be applied. In the case of conservative biochemical mechanisms the existence of a positive equilibrium in the level set $\omega_{c_{0}}(10)$ is always guaranteed. If multiple regular equilibria exist (see Sec. 5 ) in some level set $\omega_{c_{0}}$ for some values of the rate constants, then the number of equilibria is always odd.

For large mechanisms with many species and reactions the package GraTeLPy [19] can be used to search for critical fragments (of order equal to the rank of the stoichiometric matrix), that are necessary for multistationarity.

Other related graph-theoretic conditions for multistationarity have been developed recently. In the work of Craciun and Feinberg the undirected species-reaction (SR) graph is used and a graph-theoretic condition that precludes multistationarity in open system 


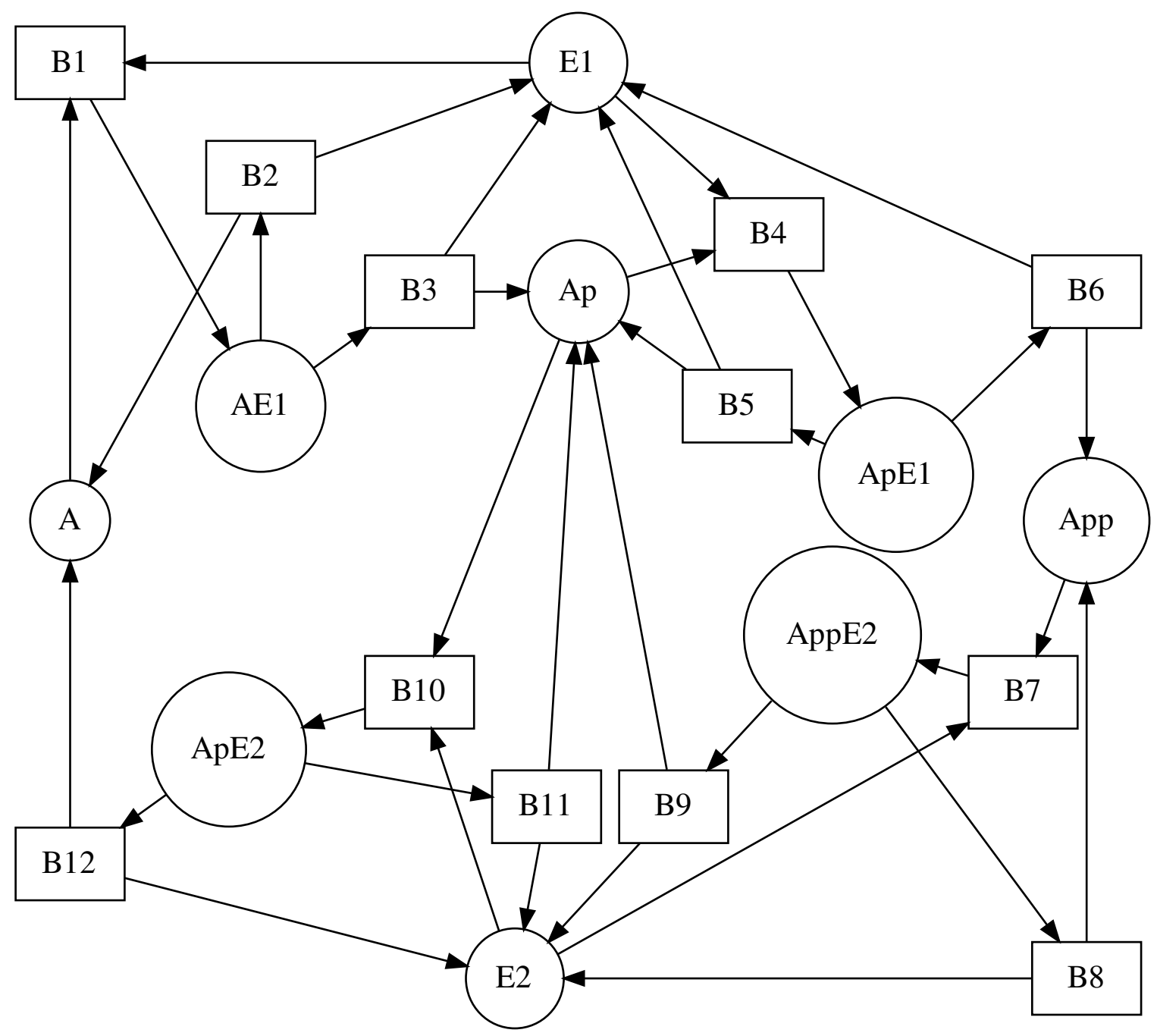

Figure 5: Bipartite digraph of the single-layer MAPK network. Reproduced from [19] under Open Access License Agreement.

mass-action kinetics models for any parameter values is obtained [7]. Banaji and Craciun obtain graph-theoretic conditions for injectivity and uniqueness of equilibria regardless of parameter values in chemical kinetics models using the SR graph [2]. In an earlier work the same authors use a signed, directed, labeled, bipartite multigraph, termed the "DSR graph" to obtain a graph-theoretic condition that rules out multiple equilibria of general interaction networks models [1].

In [13 degree theory is used to study the number of equilibria of ecological differential 
equations where $\dot{x}_{i}=x_{i} f_{i}(x)$ for all $i$. Degree theory methods have also been used in 8 to determine the number of equilibria for complex biochemical reaction networks. Degree theory arguments are used to find parameter values (rate constants and total concentrations) such that the MAPK model (37) has three equilibria or a single equilibria in [5].

\section{References}

[1] M. Banaji and G. Craciun. Graph-theoretic approaches to injectivity and multiple equilibria in systems of interacting elements. Comm. in Math. Sci., 7(4):867-900, 2009.

[2] M. Banaji and G. Craciun. Graph-theoretic criteria for injectivity and unique equilibria in general chemical reaction systems. Adv. in Appl. Math., 44(2):168-184, 2010.

[3] C. Conradi and D. Flockerzi. Multistationarity in mass action networks with applications to ERK activation. J. of Math. Biol., 65(1):107-156, 2012.

[4] C. Conradi, D. Flockerzi, and J. Raisch. Multistationarity in the activation of a mapk: Parametrizing the relevant region in parameter space. Math. Biosc., 211(1):105-131, 2008.

[5] C. Conradi and M. Mincheva. Catalytic constants enable the emergence of bistability in dual phosphorylation. J. of The Royal Soc. Interface, 11(95):20140158, 2014.

[6] C. Conradi, J. Saez-Rodriguez, E. Gilles, and J. Raisch. Using Chemical Reaction Network Theory to discard a kinetic mechanism hypothesis. Systems Biology, IEE Proceedings (now IET Systems Biology), 152(4):243-248, 2005.

[7] G. Craciun and M. Feinberg. Multiple equilibria in complex chemical reaction networks. II. SIAM J. Appl. Math., 66(4):1321-1338, 2006.

[8] G. Craciun, J. Helton, and R. Williams. Homotopy methods for counting reaction network equilibria. Math. Biosc., 216(2):140-149, 2008.

[9] P. De Leenheer, D. Angeli, and E. D. Sontag. Monotone chemical reaction networks. J. of Math. Chem., 41(3):295-314, 2007.

[10] K. Deimling. Nonlinear functional analysis. Courier Dover Publications, 2010.

[11] F. Gantmacher. Theory of Matrices. 2V. Chelsea Publishing Company, 1960.

[12] F. Harary. Graph theory. Addison-Wesley, Reading, MA, 1969. 
[13] J. Hofbauer. An index theorem for dissipative semiflows. Rocky Mountain J. of Math., 20(4):1017-1031, 1990.

[14] B. Joshi and A. Shiu. Simplifying the jacobian criterion for precluding multistationarity in chemical reaction networks. SIAM J. Appl. Math., 72(3):857-876, 2012.

[15] N. I. Markevich, J. B. Hoek, and B. N. Kholodenko. Signaling switches and bistability arising from multisite phosphorylation in protein kinase cascades. J. Cell Biol., 164(3):353-359, 2004.

[16] M. Mincheva and M. R. Roussel. Graph-theoretic methods for the analysis of chemical and biochemical networks. I. J. Math. Biol., 55(1):61-86, 2007.

[17] E. M. Ozbudak and et al. Multistability in the lactose utilization network of escherichia coli. Nature, 427(6976):737-740, 2004.

[18] A Volpert and A Ivanova. Mathematical modeling (in russian), 1987.

[19] G. Walther, M. Hartley, and M. Mincheva. Gratelpy: graph-theoretic linear stability analysis. BMC Systems Biology, 8(1):22, 2014. 


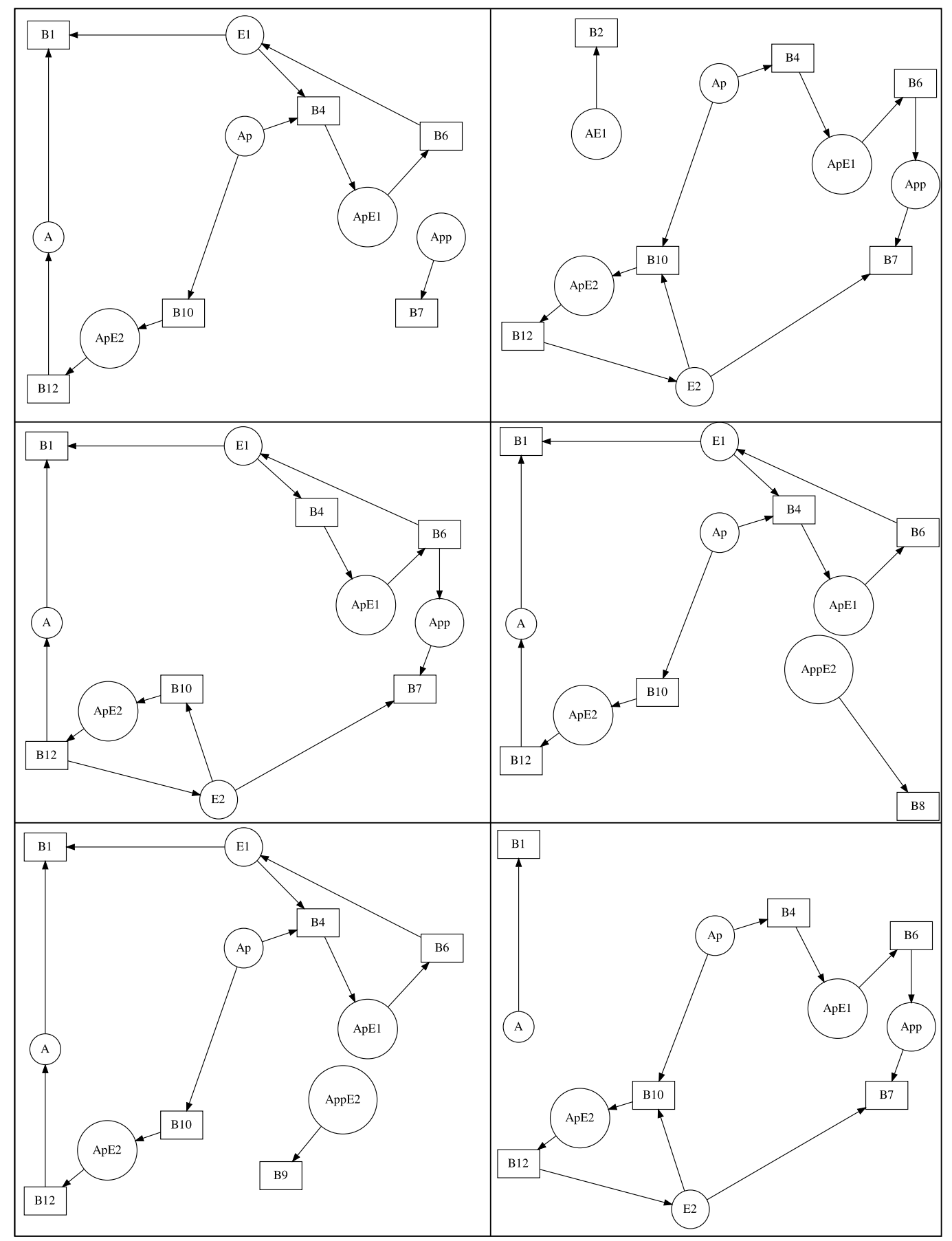

Figure 6: Critical fragments of the single-layer MAPK network found by GraTeLPy. Reproduced from [19] under Open Access License Agreement. 


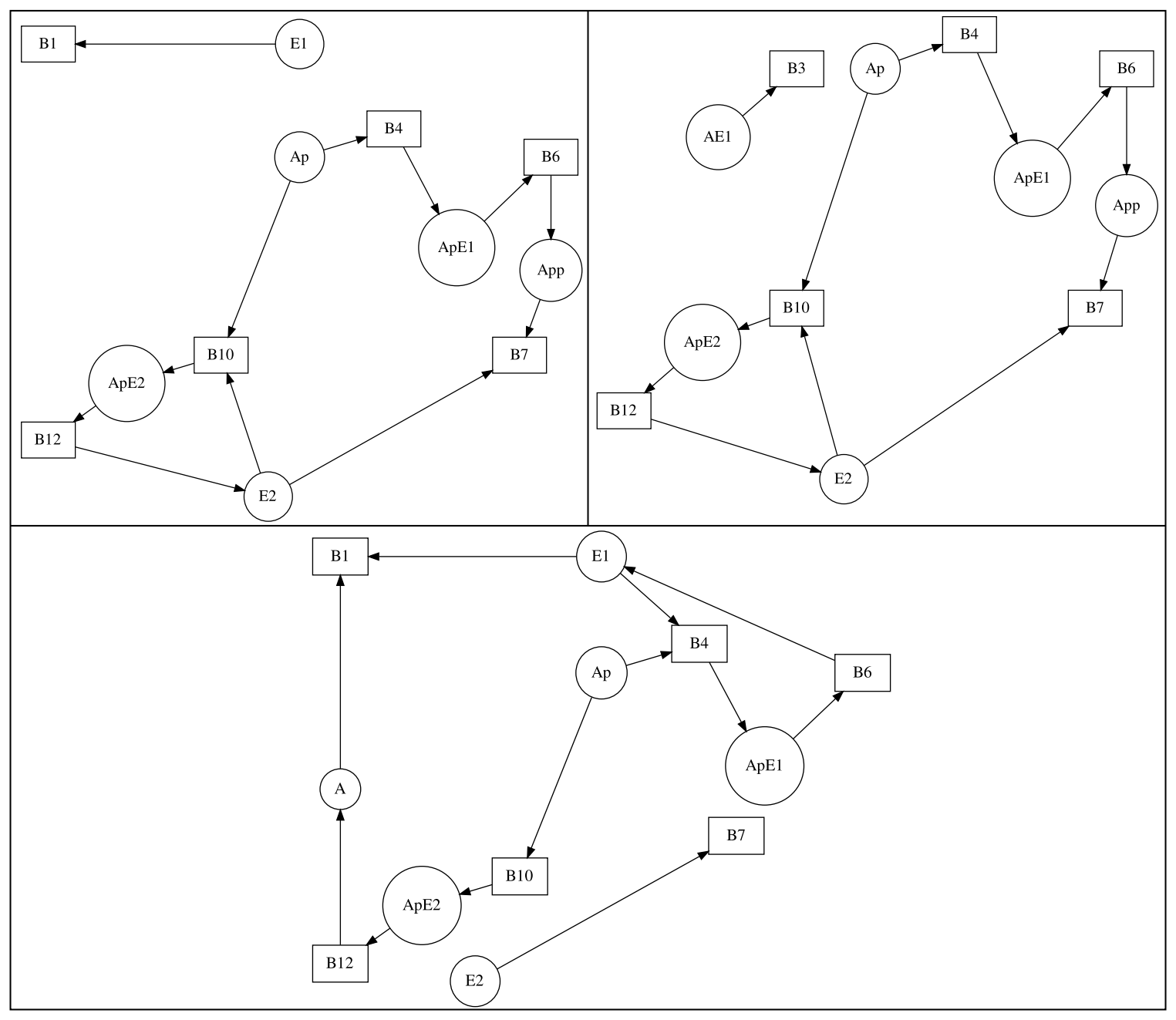

Figure 7: Critical fragments of the single-layer MAPK network found by GraTeLPy. Reproduced from [19] under Open Access License Agreement. 